Research Article

\title{
Green Synthesis of Spherical Calcium Hydroxide Nanoparticles in the Presence of Tannic Acid
}

\author{
Peiyuan Chen $\left(\mathbb{D}\right.$, Yonghui Wang $\mathbb{D}^{D}$, Shicheng He $\mathbb{D}$, Pengju Wang $\mathbb{D}^{\circ}$, Ying Xu $(\mathbb{D}$, \\ and Liheng Zhang (iD \\ School of Civil Engineering and Architecture, Anhui University of Science and Technology, Huainan 232001, China \\ Correspondence should be addressed to Peiyuan Chen; peiyuan29@126.com
}

Received 28 April 2020; Revised 24 September 2020; Accepted 25 September 2020; Published 10 October 2020

Academic Editor: Pietro Russo

Copyright (c) 2020 Peiyuan Chen et al. This is an open access article distributed under the Creative Commons Attribution License, which permits unrestricted use, distribution, and reproduction in any medium, provided the original work is properly cited.

\begin{abstract}
This study proposes a green, low-cost, and robust method to synthesize spherical calcium hydroxide (CH) nanoparticles based on the coprecipitation of $\mathrm{Ca}^{2+}$ and $\mathrm{OH}^{-}$in the presence of tannic acid (TA), an inexpensive plant-derived polyphenol. It has been found that the morphology and crystalline phase of the produced $\mathrm{CH}$ nanoparticles can be controlled by TA. With the increasing dosage of TA, the crystallinity of the $\mathrm{CH}$ particles reduces, changing from well crystallization to amorphous phases. Experimental evidence suggests that complexion reaction took place between $\mathrm{TA}$ and $\mathrm{Ca}^{2+}$, which gave the $\mathrm{Ca}^{2+}$ a strong bond effect. As a result, the nucleation and growth of $\mathrm{CH}$ were significantly affected. $\mathrm{CH}$ nanoparticles with sizes of about $50 \mathrm{~nm}$ have been successfully synthesized by using $0.3 \%$ or more TA.
\end{abstract}

\section{Introduction}

Calcium hydroxide $(\mathrm{CH})$ nanoparticle has gained extensive attentions as an eco-friendly material $[1,2]$ with numerous applications in the natural stone, wood, and cultural heritage conservation [2-6], wall painting $[7,8]$, and consolidation of art works [9], as well as the treatment of catalyst and adsorbents $[10,11]$. Although great efforts have been made on the synthesis approaches of such nanoparticle, conventional precipitation process hardly works due to its moderate solubility $[1,7]$. The assistance of high temperature and high pressure or organic solvents is to some content essential to synthesis desired $\mathrm{CH}$ nanoparticles [1]. Many methods have been proposed to synthesize $\mathrm{CH}$ nanoparticles, including the homogeneous hydrolytic reactions [12], water-in-oil microemulsion [13], hydrogen plasma-metal reaction [7], sonochemical method, and wet chemical approaches $[14,15]$. Nevertheless, more environmentally friendly and less expensive method is always more desirable for $\mathrm{CH}$ nanoparticle production [2].

Recently, we found that tannic acid (TA), an inexpensive plant-derived polyphenol, can be used as an effective surfactant to stabilize and facilitate the production of $\mathrm{CH}$ nanoparticles through a co-precipitation process at ambient temperature and pressure. TA was noticed because it not only is inexpensive and available worldwide unlimited [16] but also possesses the prominent functional properties in terms of antioxidant, antimutagenic, anti-inflammatory, biodegradability, and antitumour activity $[17,18]$. TA is molecularly composed of a central glucose core and several gallic acid monomers. The benzene hydroxyl groups within TA molecular structure give TA the inherent bonding capacity with metal cations, such as $\mathrm{Ca}^{2+}$ through complexion reaction $[19,20]$. Therefore, the nucleation and growth of $\mathrm{CH}$ crystals in the co-precipitation system is assumed to be influenced, decelerating the growth speeds of $\mathrm{CH}$ crystals, which may help achieve $\mathrm{CH}$ precipitates in nanosizes.

Therefore, this paper was conducted to investigate the effect of TA on the morphology and crystal form of $\mathrm{CH}$ nanoparticles in a co-precipitation process using $\mathrm{CaCl}_{2}$ and $\mathrm{NaOH}$. To this end, $\mathrm{Ca}^{2+}$ was firstly mixed with TA solution to produce sufficient complexion. Then, $\mathrm{NaOH}$ was added as the precipitator to produce $\mathrm{CH}$ precipitates. Field-emission scanning electron microscopy (SEM), transmission electron microscope (TEM), and Brunauer, Emmett, and Teller (BET) surface area analyzer were applied to characterize the morphological and geometric properties of the $\mathrm{CH}$ precipitates. X-ray diffractometer (XRD) and Fourier transform 


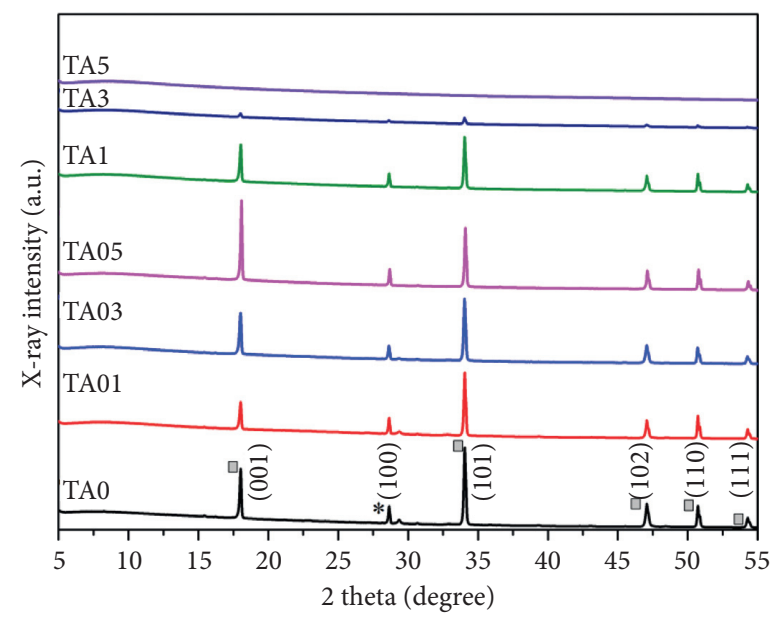

口 $\mathrm{CH}$

* Calcite

FIGURE 1: XRD patterns of $\mathrm{CH}$ precipitates with different dosages of TA.

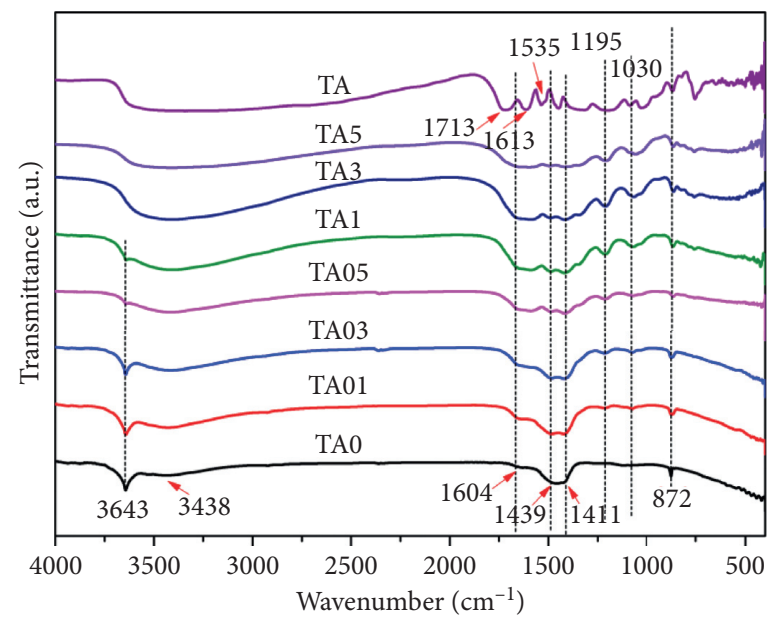

Figure 2: FTIR spectra of $\mathrm{CH}$ precipitates and TA.

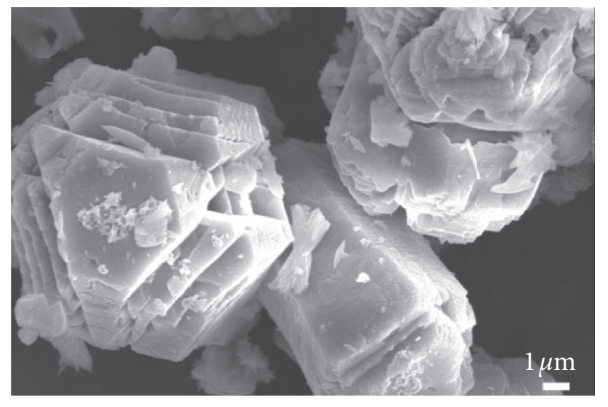

(a)

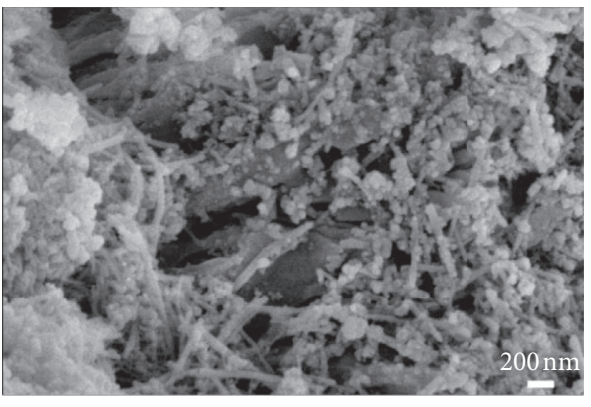

(b)

Figure 3: Continued. 


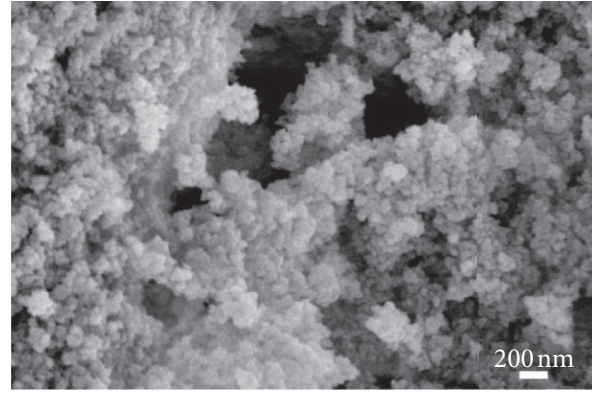

(c)

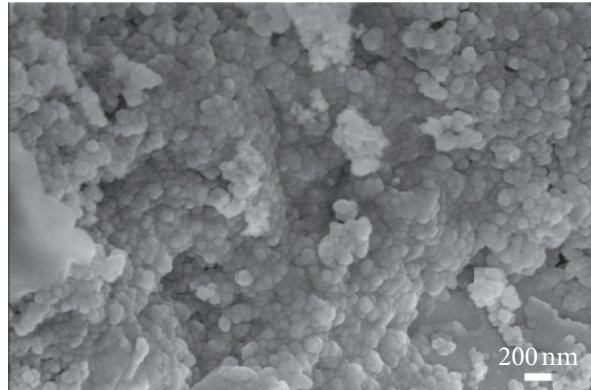

(e)

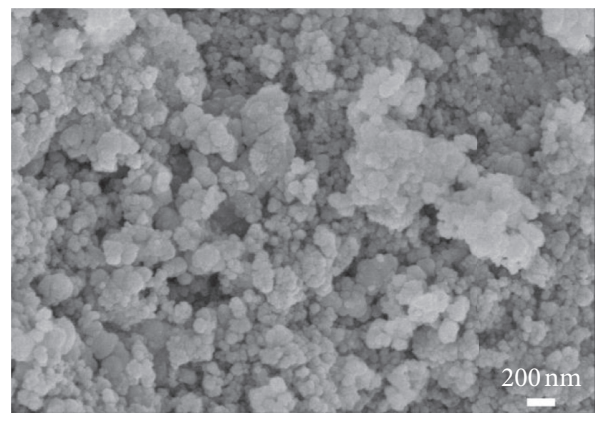

(g)

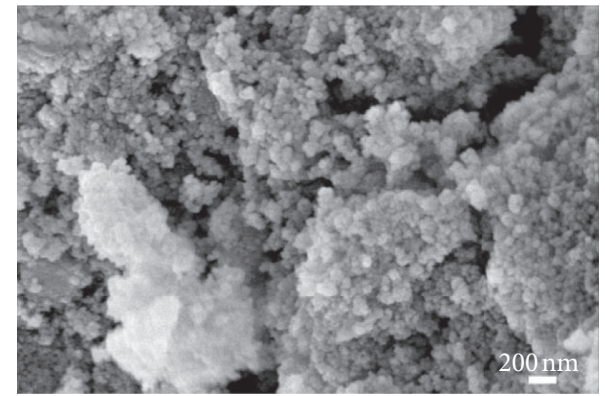

(d)

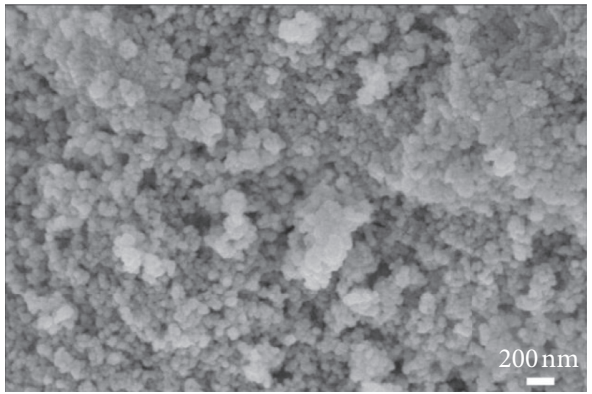

(f)

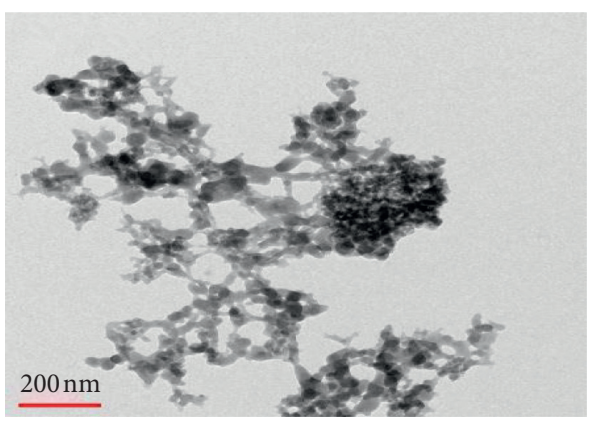

(h)

Figure 3: Micrograms of CH precipitates. SEM images of (a) TA0, (b) TA01, (c) TA03, (d) TA05, (e) TA1, (f) TA3, and (g) TA5 and (h) TEM image of TA05.

infrared spectrometer (FTIR) were used to study the morphological and crystal features of $\mathrm{CH}$. Mechanism regarding the formation of $\mathrm{CH}$ nanoparticles was discussed to understand the effect of TA on the nucleation and growth of $\mathrm{CH}$ particles.

\section{Experimental Study}

2.1. Materials and Methods. $\mathrm{CaCl}_{2}, \mathrm{NaOH}$, and isopropanol of analytical purity were purchased from Sinopharm Chemical Reagent Co., Ltd. and were used as received without further purification. Analytically, pure TA was provided by Aladdin Industrial Corporation. Double deionized water was used throughout this study. The proposed synthesis route of $\mathrm{CH}$ was simply complied with a conventional co-precipitation process expressed in

$$
\mathrm{Ca}^{2+}+2 \mathrm{OH}^{-} \longrightarrow \mathrm{Ca}(\mathrm{OH})_{2}(\mathrm{~s})
$$

The dosage of TA was examined as the experimental variable to understand its effect on the morphology and the crystal form of $\mathrm{CH}$ precipitates. To this end, TA solutions with mass concentration of $0 \%, 0.01 \%, 0.03 \%, 0.05 \%, 0.1 \%$, $0.3 \%$, and $0.5 \%$ were made by adding TA with $100 \mathrm{ml}$ double deionized water. These solutions were sealed by plastics immediately after which TA had been agitated by glass rods to be completely dissolved. Then, $2.2 \mathrm{~g} \mathrm{CaCl}_{2}$ was added into TA solutions and was vigorously stirred. These solutions were sealed and statically placed for 1 hour to ensure a sufficient complexion reaction to take place between TA and $\mathrm{Ca}^{2+}$. Subsequently, $100 \mathrm{ml} 0.2 \mathrm{M} \mathrm{NaOH}$ was added into the aged solutions slowly. The mixed solutions were then sealed and stirred for another 1 hour by a magnetic stirrer at the speed of $50 \mathrm{rpm}$. The final concentrations of both the $\mathrm{CaCl}_{2}$ and $\mathrm{NaOH}$ in the solutions were the same at $0.1 \mathrm{M}$. At the predetermined time, about $300 \mathrm{ml}$ isopropanol was poured into the solutions aiming to cease the nucleation or growth 


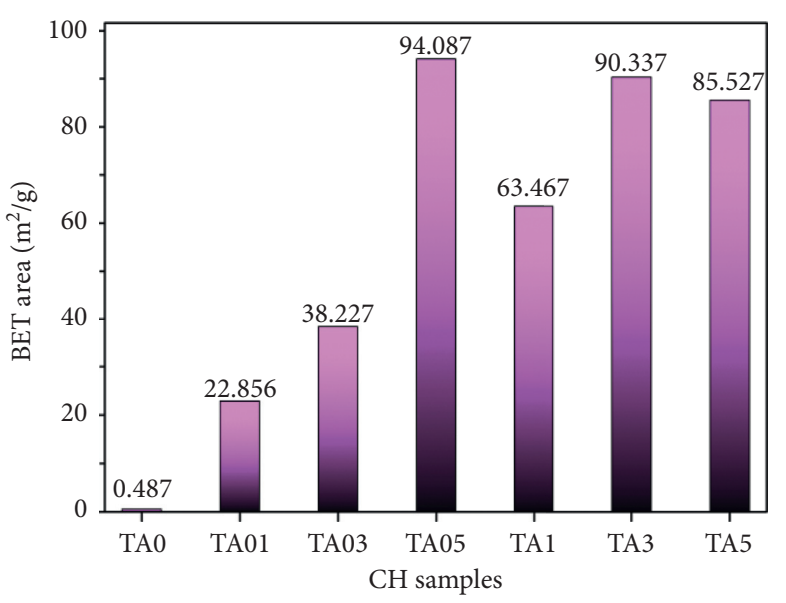

Figure 4: BET area of $\mathrm{CH}$ precipitates.

of $\mathrm{CH}$ crystals. The precipitates were vacuum filtrated and washed by isopropanol two times and double deionized water three times. Finally, the precipitates were vacuum dried for 24 hours and stored in vacuum plastic bags prior to the characterization analysis. These synthesized $\mathrm{CH}$ precipitates were coded as TA0, TA01, TA03, TA05, TA1, TA3, and TA5, respectively, based on the percentage concentration of the used TA solutions.

2.2. Characterization Methods. The morphology of the synthesized $\mathrm{CH}$ particles was studied by a FlexSEM 1000 SEM. Platinum particles sputtering for $120 \mathrm{~s}$ was performed to gain well electrical conductivity of the samples. A JEM2100F TEM was applied to further examine the morphology of the produced $\mathrm{CH}$ nanoparticles. XRD was conducted to investigate the crystalline forms of the $\mathrm{CH}$ samples by using a TTR-III h/h rotating anode X-ray diffractometer with $\mathrm{Cu}$ Ka radiation at $40 \mathrm{kV}$ and $200 \mathrm{~mA}$. The scan speed and scope were, respectively, $8^{\circ} / \mathrm{min}$ and $2 \theta=5^{\circ}-55^{\circ}$ for each specimen. Nicolet iS50 FTIR was used to further determine the composition of the $\mathrm{CH}$ samples. BET surface area of $\mathrm{CH}$ samples was also analyzed using a Micromeritics Tristar II $3020 \mathrm{M}$.

\section{Results and Discussion}

The effect of TA on the crystal form of $\mathrm{CH}$ precipitates was investigated by XRD, as presented in Figure 1. Both crystalline and amorphous $\mathrm{CH}$ have been identified along with a small amount of calcite, which was produced by the slight carbonation of $\mathrm{CH}$ samples. In general, the nucleation and growth of $\mathrm{CH}$ crystals have been prominently affected by TA molecules. The characteristic peak heights of $\mathrm{CH}$ precipitates at $2 \theta=34^{\circ}$ are found to decrease or even disappear in TA5 with increasing dosages of TA. In addition to that the residual peaks are hardly detected in TA3 and completely disappear in TA5. This suggests that the existence of TA interfered the crystallization of $\mathrm{CH}$, resulting $\mathrm{CH}$ with poor crystallinity when more TA has been added, especially amorphous $\mathrm{CH}$ when $0.5 \%$ TA was used.
FTIR was applied to further reveal the role of TA in formation of $\mathrm{CH}$, as shown in Figure 2. The absorption band at $3643 \mathrm{~cm}^{-1}$ belongs to the $\mathrm{O}-\mathrm{H}$ stretching in the $\mathrm{CH}$ crystal $[7,21]$. The minor absorption bands at $872 \mathrm{~cm}^{-1}$ and $1411-1439 \mathrm{~cm}^{-1}$ correspond to the symmetric and asymmetric stretching of $\mathrm{CO}_{3}$ group of calcite [7], indicating the slight carbonation of $\mathrm{CH}$, which agrees with the XRD result. The absorption bands at 3438 and $1604 \mathrm{~cm}^{-1}$ are attributed to the $\mathrm{O}-\mathrm{H}$ stretching and bending vibrations, respectively, implying that physisorbed $\mathrm{H}_{2} \mathrm{O}$ molecules are linked to $\mathrm{CH}$ crystals [2]. Moreover, the absorption bands of TA at 1713, $1613,1535,1195$, and $1030 \mathrm{~cm}^{-1}$ correspond to $\mathrm{C}=\mathrm{C}$ stretch of benzene ring $\left(1713 \mathrm{~cm}^{-1}\right), \mathrm{C}-\mathrm{C}$ stretch and $\mathrm{C}-\mathrm{H}$ deformation in plane of benzene ring $\left(1613 \mathrm{~cm}^{-1}\right), \mathrm{C}=\mathrm{O}$ stretch of carboxylic acid $\left(1535 \mathrm{~cm}^{-1}\right),(\mathrm{O}-\mathrm{H})$ deformation in plane of phenolic and carboxylic acid, (C-C) stretch and $(\mathrm{C}-\mathrm{H})$ deformation in plane of benzene ring $\left(1195 \mathrm{~cm}^{-1}\right)$, and $(\mathrm{C}=\mathrm{O})$ stretch of carboxylic acid $\left(1030 \mathrm{~cm}^{-1}\right)$ [22]. The absorption bands at 1195 and $1030 \mathrm{~cm}^{-1}$ were detected in $\mathrm{CH}$ samples of TA01 to TA05, suggesting the existence of residual TA in $\mathrm{CH}$ samples.

With the increasing dosage of TA, the absorption band at $3643 \mathrm{~cm}^{-1}$ of the $\mathrm{CH}$ precipitate decreases or disappears in TA3 and TA5, displaying a similar tendency to that of XRD results. This further confirms the impeding effect of TA on crystallization of $\mathrm{CH}$. In addition, more distinct absorption peaks at $1604 \mathrm{~cm}^{-1}$ can be found when more TA has been used. This may indicate that $\mathrm{CH}$ with poor crystallinity contains physisorbed $\mathrm{H}_{2} \mathrm{O}$ molecules in its structure.

Figure 3 shows the micrographs of $\mathrm{CH}$ precipitates by SEM and TEM. These figures clearly demonstrate that TA also exerted significant influence on the morphology of $\mathrm{CH}$ particles. The control group TA0 shown in Figure 3(a) displays prismatic microscale particles with much dense structure. This is a kind of classic morphology of the hexagonal $\mathrm{CH}$ crystal. When TA has been added, the $\mathrm{CH}$ particle becomes porous in structure. Both nanosized fiberous and spherical particles are observed, which acts as a coating layer of the dense matrix, as can be found in Figure 3(b). This is consistent with the results of XRD and FTIR as $\mathrm{CH}$ is in poor crystallization form when TA was presented and therefore no hexagonal $\mathrm{CH}$ was observed. When more TA has been added (Figures 3(c)-3(g), spherical $\mathrm{CH}$ nanoparticles are formed instead of the fiberous $\mathrm{CH}$ nanoparticles or dense micron-scale $\mathrm{CH}$ particles. These spherical $\mathrm{CH}$ nanoparticles have fairly homogeneous sizes of about $50 \mathrm{~nm}$, some of which adhere to each other, which can be further confirmed by the TEM image of TA05 in Figure $3(\mathrm{~h})$ as the representative.

One of the direct benefits of producing $\mathrm{CH}$ nanoparticles with TA solution is the enlarged BET area, as charted in Figure 4. The development tendency of the BET areas of all $\mathrm{CH}$ samples generally complies to a paraboliclike trend. The BET area of TA0 is just $0.487 \mathrm{~m}^{2} / \mathrm{g}$; nevertheless, it increases sharply to the maximum $94.087 \mathrm{~m}^{2} /$ g of TA05 samples, which is about 193 times larger than that of TA0. Slight reduced BET areas as compared to that of TA05 are found in $\mathrm{CH}$ samples incorporated with more TA than $0.05 \%$. 


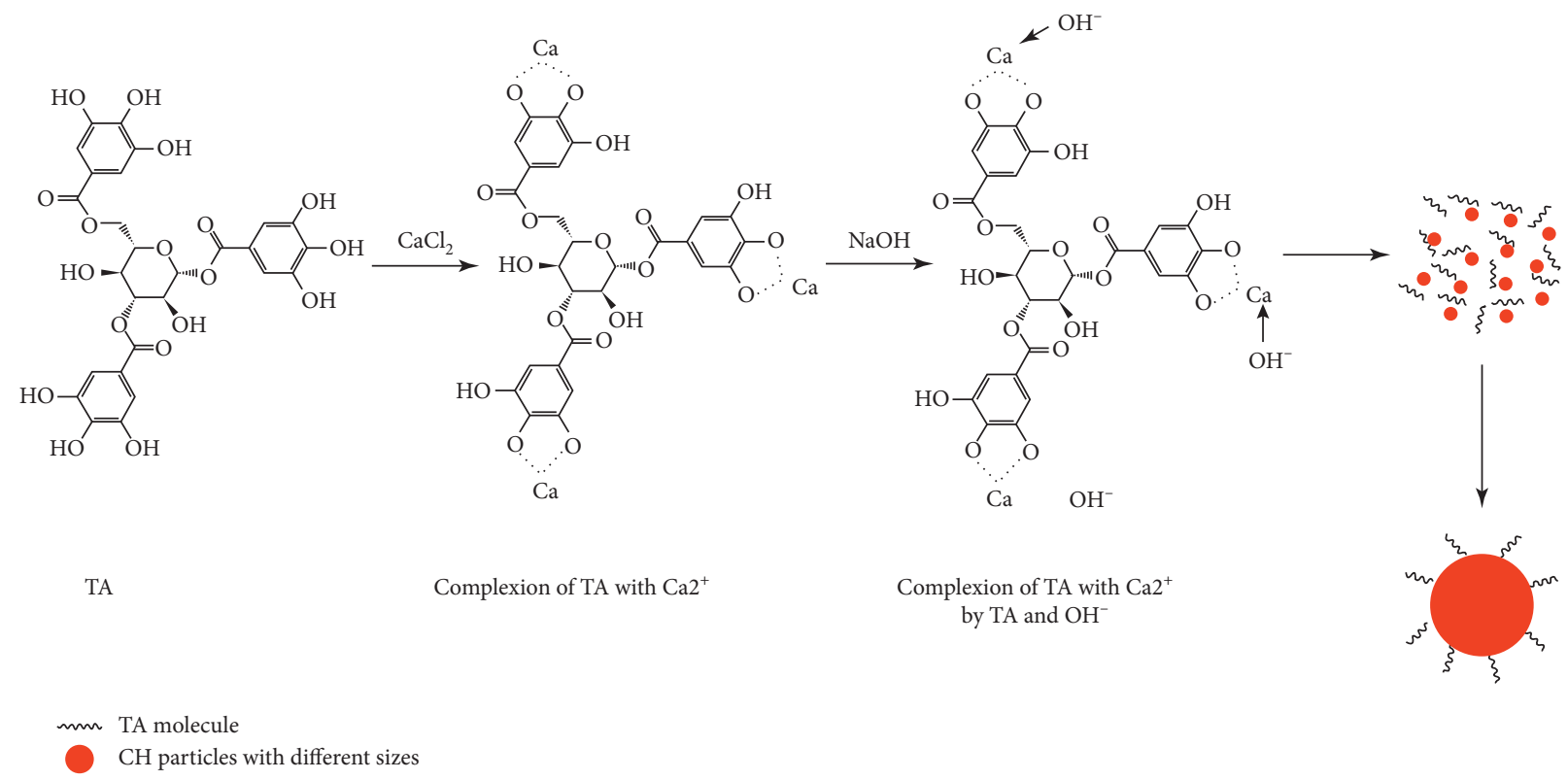

FIgURE 5: Schematic of the formation of $\mathrm{CH}$ nanoparticles with the assistant of TA.

Based on the above analysis, the formation of $\mathrm{CH}$ nanoparticles in the presence of TA can be viewed as a fourstep process. Firstly, the phenolic hydroxyl groups in the TA molecule capture $\mathrm{Ca}^{2+}$ through a complexion reaction. $\mathrm{Ca}^{2+}$ is bound to two adjacent oxygen atoms. This process is crucial for the formation of $\mathrm{CH}$ nanoparticles. Normally, without $\mathrm{TA}, \mathrm{Ca}^{2+}$ and $\mathrm{OH}^{-}$in a co-precipitation system react freely to nucleate and grow into insoluble $\mathrm{CH}$ particles, as shown in Figure 5. Nevertheless, with the presence of TA, $\mathrm{Ca}^{2+}$ are bound to TA molecules. Consequently, the reaction between $\mathrm{Ca}^{2+}$ and $\mathrm{OH}^{-}$is hindered due to the competitive binding between TA and $\mathrm{OH}^{-}$to $\mathrm{Ca}^{2+}$. The binding of $\mathrm{Ca}^{2+}$ and $\mathrm{OH}^{-}$thus become more difficult due to the bonding of $\mathrm{Ca}^{2+}$ by TA. In this way, the newly formed $\mathrm{CH}$ nucleus is much more stable, decelerating the nucleation process. As a result, the growth of $\mathrm{CH}$ nanoparticles is inhibited, leading to nanosized $\mathrm{CH}$ particles with poor crystallinity.

It is of great importance to control the crystallinity and morphology of the $\mathrm{CH}$ precipitates since it is a major hydration products of Portland cement. Not only compacted microstructure can be gained but also a series of synergistic binding effect between $\mathrm{CH}$ and other hydration products, i.e., calcium silicate hydrate (CSH) gel may be triggered due to the poor crystallinity of the $\mathrm{CH}$ in the presence of TA. This will be further investigated in our ongoing studies.

\section{Conclusions}

A green, low-cost, robust, and simple synthesis method for $\mathrm{CH}$ nanoparticles has been proposed. This method uses TA as the surfactant through a co-precipitation method of $\mathrm{Ca}^{2+}$ and $\mathrm{OH}^{-}$under ambient temperature and pressure. It was found that TA played a pivotal role in controlling both the morphology and crystallization of the produced $\mathrm{CH}$ nanoparticles. With the increasing dosage of TA, the crystallinity of $\mathrm{CH}$ tended to be poorer to even the amorphous phase. Meanwhile, $\mathrm{CH}$ nanoparticles with sizes of about $50 \mathrm{~nm}$ have been successfully synthesized in groups containing $0.3 \%$ TA or more. This is because TA in the coprecipitation system not only acted as an ions capturer to hinder the reaction between $\mathrm{Ca}^{2+}$ and $\mathrm{OH}^{-}$but also functioned as a layer of protective coating, leading to decelerated nucleation and growth of $\mathrm{CH}$ particles. This study may provide a useful hint on the controlling of the morphology and crystallinity of the $\mathrm{CH}$ during cement hydration so that the properties of hardened cementitious composites can be tuned.

\section{Data Availability}

Data used to support the findings of the study are available from the corresponding author upon request.

\section{Conflicts of Interest}

The authors declare no conflicts of interest.

\section{Acknowledgments}

This work was supported the China Postdoctoral Science Foundation (no. 2018M632518), University Natural Science Research Project of Anhui Province (KJ2018A0074), Natural Science Foundation of Anhui Province (1908085QE213), and Key Research and Development Program Project of Anhui Province (201904a07020081).

\section{References}

[1] M. D. Khan, J. W. Ahn, and G. Nam, "Environmental benign synthesis, characterization and mechanism studies of green calcium hydroxide nano-plates derived from waste oyster 
shells," Journal of Environmental Management, vol. 223, pp. 947-951, 2018.

[2] M. Darroudi, M. Bagherpour, H. A. Hosseini, and M. Ebrahimi, "Biopolymer-assisted green synthesis and characterization of calcium hydroxide nanoparticles," $\mathrm{Ce}$ ramics International, vol. 42, no. 3, pp. 3816-3819, 2016.

[3] C. Rodriguez-Navarro, A. Suzuki, and E. Ruiz-Agudo, "Alcohol dispersions of calcium hydroxide nanoparticles for stone conservation," Langmuir, vol. 29, no. 36, pp. 11457-11470, 2013.

[4] V. Daniele and G. Taglieri, "Synthesis of $\mathrm{Ca}(\mathrm{OH})_{2}$ nanoparticles with the addition of Triton X-100. Protective treatments on natural stones: preliminary results," Journal of Cultural Heritage, vol. 13, no. 1, pp. 40-46, 2012.

[5] R. Giorgi, D. Chelazzi, and P. Baglioni, "Nanoparticles of calcium hydroxide for wood conservation. The deacidification of the Vasa warship," Langmuir: the ACS Journal of Surfaces and Colloids, vol. 21, no. 23, p. 10743, 2005.

[6] G. Poggi, N. Toccafondi, D. Chelazzi, P. Canton, R. Giorgi, and P. Baglioni, "Calcium hydroxide nanoparticles from solvothermal reaction for the deacidification of degraded waterlogged wood," Journal of Colloid and Interface Science, vol. 473, pp. 1-8, 2016.

[7] T. Liu, Y. Zhu, X. Zhang, T. Zhang, T. Zhang, and X. Li, "Synthesis and characterization of calcium hydroxide nanoparticles by hydrogen plasma-metal reaction method," $M a-$ terials Letters, vol. 64, no. 23, pp. 2575-2577, 2010.

[8] R. Giorgi, M. Ambrosi, N. Toccafondi, and P. Baglioni, "Nanoparticles for cultural heritage conservation: calcium and barium hydroxide nanoparticles for wall painting consolidation," Chemistry-A European Journal, vol. 16, no. 31, pp. 9374-9382, 2010.

[9] P. Baglioni, D. Chelazzi, R. Giorgi, E. Carretti, N. Toccafondi, and Y. Jaidar, "Commercial $\mathrm{Ca}(\mathrm{OH})_{2}$ nanoparticles for the consolidation of immovable works of art," Applied Physics A, vol. 114, no. 3, pp. 723-732, 2014.

[10] R. S. Boynton, Chemistry and Technology of Lime and Limestone, John Wiley \& Sons, Hoboken, NJ, US, 1980.

[11] A. Samanta, S. Podder, C. K. Ghosh et al., "ROS mediated high anti-bacterial efficacy of strain tolerant layered phase pure nano-calcium hydroxide," Journal of the Mechanical Behavior of Biomedical Materials, vol. 72, pp. 110-128, 2017.

[12] M. Ambrosi, L. Dei, R. Giorgi, C. Neto, and P. Baglioni, "Colloidal particles of $\mathrm{Ca}(\mathrm{OH})_{2}$ : properties and applications to restoration of frescoes," Langmuir, vol. 17, no. 14, pp. 4251-4255, 2001.

[13] B. Salvadori and L. Dei, "Synthesis of $\mathrm{Ca}(\mathrm{OH})_{2}$ nanoparticles from diols," Langmuir, vol. 17, no. 8, pp. 2371-2374, 2001.

[14] H. R. Momenian, S. Gholamrezaei, M. Salavati-Niasari, B. Pedram, F. Mozaffar, and D. Ghanbari, "Sonochemical synthesis and photocatalytic properties of metal hydroxide and carbonate (M:Mg, Ca, Sr or Ba) nanoparticles," Journal of Cluster Science, vol. 24, no. 4, pp. 1031-1042, 2013.

[15] A. Roy and J. Bhattacharya, "Synthesis of $\mathrm{Ca}(\mathrm{OH})_{2}$ nanoparticles by wet chemical method," Micro \& Nano Letters Journal, vol. 5, no. 2, pp. 131-134, 2010.

[16] D. Huang, Z. Zhang, Z. Ma, Q. Quan, and J. Ding, “A facile strategy for the modification of chitosan/gliadin composites by tannic acid," Materials Letters, vol. 240, pp. 209-212, 2019.

[17] M. L. Picchio, Y. G. Linck, G. A. Monti, L. M. Gugliotta, R. J. Minari, and C. I. Alvarez Igarzabal, "Casein films crosslinked by tannic acid for food packaging applications," Food Hydrocolloids, vol. 84, pp. 424-434, 2018.
[18] W. Xu, E.-H. Han, and Z. Wang, "Effect of tannic acid on corrosion behavior of carbon steel in $\mathrm{NaCl}$ solution," Journal of Materials Science \& Technology, vol. 35, no. 1, pp. 64-75, 2019.

[19] L. Coppola, D. Coffetti, and E. Crotti, "Use of tartaric acid for the production of sustainable portland-free CSA-based mortars," Construction and Building Materials, vol. 171, pp. 243-249, 2018.

[20] C. Ringwald and V. Ball, "Layer-by-layer deposition of tannic acid and $\mathrm{Fe}^{3+}$ cations is of electrostatic nature but almost ionic strength independent at $\mathrm{pH}$ 5," Journal of Colloid and Interface Science, vol. 450, pp. 119-126, 2015.

[21] N. Asikin-Mijan, Y. H. Taufiq-Yap, and H. V. Lee, "Synthesis of clamshell derived $\mathrm{Ca}(\mathrm{OH})_{2}$ nano-particles via simple surfactant-hydration treatment," Chemical Engineering Journal, vol. 262, pp. 1043-1051, 2015.

[22] L. Wang, J. Wang, X. Qian, Y. Fang, P. Chen, and A. Tuinukuafe, "Tea stain-inspired treatment for fine recycled concrete aggregates," Construction and Building Materials, vol. 262, Article ID 120027, 2020. 